\title{
Esophageal Food Impaction: A Retrospective Chart Review
}

\author{
Dhineshreddy Gurala ${ }^{\mathrm{a}}$, Abhishek Polavarapu ${ }^{\mathrm{b}}$, Jobin Philipose ${ }^{\mathrm{b}, \mathrm{f}}$, Shivantha Amarnath ${ }^{\mathrm{a}}$, \\ Akshay Avula ${ }^{c}$, Pretty Sara Idiculla ${ }^{d}$, Seleshi Demissie ${ }^{e}$, Vivek Gumaste ${ }^{b}$
}

\begin{abstract}
Background: Esophageal food impaction (EFI) is the third most common non-biliary emergency in gastroenterology, with an annual incidence rate of 13 episodes per 100,000 person-years and 1,500 deaths per year. Patients presenting with food impaction often have underlying esophageal pathology. We evaluated the possible risk factors for EFI in our study.
\end{abstract}

Methods: We performed a retrospective chart review of 455 patients at Staten Island University Hospital (SIUH) that presented with symptoms of food impaction from 1999 to 2017. We analyzed relevant clinical data such as age, risk factors, type of food bolus, location, administration of glucagon, endoscopic technique and complications.

Results: Overall, 174 patients had endoscopically confirmed EFI. The majority were males 102/174 (58.6\%). Esophageal pathological findings included esophagitis in 58/174 (33.3\%), strictures in 43/174 (24.7\%), hiatal hernias in 29/174 (16.6\%) and Schatzki's rings in $15 / 174(8.6 \%)$. Thirty-two out of $174(18.3 \%)$ had normal endoscopic findings. Diabetes mellitus (DM) was reported in 20/174 (11.4\%) patients. The type of food impacted was mostly meat in 73/174 (41.9\%) cases. The location of EFI was mainly in the lower one-third of the esophagus in 94/174 (54\%). The endoscopic push technique was used in $95 / 174(54.5 \%)$ patients and the pull technique in $83 / 174(47.7 \%)$ cases. The endoscopic therapeutic intervention was successful as a first attempt in 165/175 (94.8\%) patients. Complications were reported in only $5 / 174(2.8 \%)$, and these mostly comprised of perforations and tears. Glucagon was given to $74 / 174$ (42.5\%) patients. The median door-to-scope time (time of presentation at the emergency

Manuscript submitted April 9, 2021, accepted April 26, 2021

Published online June 19, 2021

aDepartment of Internal Medicine, Staten Island University Hospital, Northwell Health, Staten Island, NY, USA

${ }^{b}$ Department of Gastroenterology and Hepatology, Staten Island University Hospital, Northwell Health, Staten Island, NY, USA

'Department of Pulmonary and Critical Care, Staten Island University Hospital, Northwell Health, Staten Island, NY, USA

${ }^{\mathrm{d}}$ Department of Medicine, Sree Gokulam Medical College and Research Foundation, Trivandrum, India

'Biostatistics Unit, Staten Island University Hospital, Northwell Health, Staten Island, NY, USA

${ }^{f}$ Corresponding Author: Jobin Philipose, Department of Gastroenterology and Hepatology, Staten Island University Hospital, Northwell Health, 202 Grasmere Dr, Staten Island, NY 10305, USA. Email: philipose.jobin@gmail.com

doi: https://doi.org/10.14740/gr1387 department to endoscopic intervention) was $7 \mathrm{~h}$ (range 1.5 - $24 \mathrm{~h}$ ) in patients who had received glucagon as opposed to $7 \mathrm{~h}$ (range $1-24 \mathrm{~h}$ ) in patients who did not receive it.

Conclusion: EFI is more common in males. Esophageal strictures and hiatal hernias were the most common pathologies found in endoscopy. Esophagitis was evident in $33.3 \%$ of patients, but if it was the cause or consequence of EFI is not clearly understood. DM was associated with food impaction in only $11.4 \%$ of patients, but more studies are needed to determine if DM has a stronger association with EFI. The door-to-scope time was shorter in patients who had received glucagon. Endoscopy is a safe and effective therapeutic intervention for EFI, and complications reported were minimal.

Keywords: Esophageal disorders; Upper endoscopy; Food impaction; Dysphagia; Management

\section{Introduction}

The esophagus is the most common site for foreign body impaction in the gastrointestinal (GI) tract [1]. Many objects such as coins, toys, bones and batteries can become obstructed, but food is the most common cause of esophageal food impaction (EFI) in adults [1]. EFI is the third most common non-biliary emergency in gastroenterology following upper and lower GI bleeding, with an estimated annual incidence of 13 episodes per 100,000 person-years [2] and 1,500 deaths per year [3]. EFI is frequent in adults in their fourth or fifth decades of life. Interestingly, the prevalence has increased in young adults due to the rising incidence of eosinophilic esophagitis (EoE) [4]. Risk factors for EFI include peptic strictures, Schatzki's ring and EoE to name a few. Retrospective studies have demonstrated that Schatzki's ring and peptic stricture are the most common etiologies $[4,5]$. Different studies have identified various structural and functional esophageal abnormalities that may potentially increase the risk of EFI, but these vary widely between studies. The incidence, demographic factors and etiologies of EFI are not necessarily static, especially considering the recent rise in EoE.

Intravenous glucagon is the most commonly used agent for EFI in the emergency department (ED), as it helps to relieve symptoms by relaxing the lower esophageal sphincter (LES) [6]. However, it is less effective in patients with underlying anatomical or structural abnormalities [6]. The present study aimed to evaluate potential risk factors of EFI in patients 


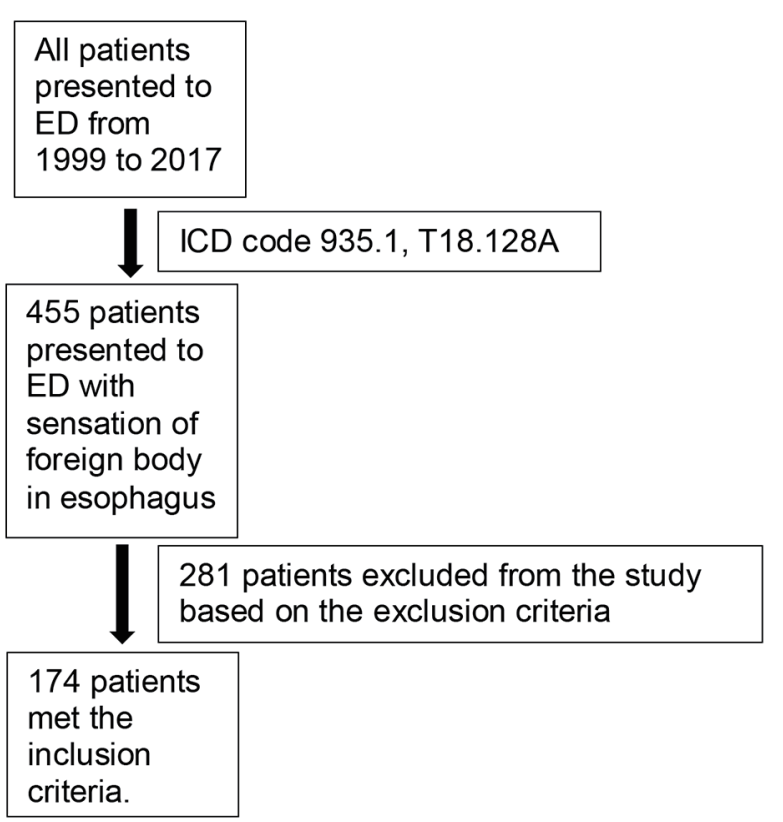

Figure 1. Final sample size including 174 patients that met our inclusion.

who presented at Staten Island University Hospital (SIUH). Additionally, we aimed to investigate the association between the use of glucagon and the door-to-scope time.

\section{Materials and Methods}

This study was approved by the Institutional Research Boards of the participating institution, and the study was conducted in compliance with the ethical standards of the responsible institution on human subjects as well as with the Helsinki Declaration.

We performed a retrospective chart review of all adult patients (18 - 80 years of age) who presented with a clinical history and symptoms suggestive of EFI to SIUH from January 1999 to November 2017 and subsequently underwent esophagogastroduodenoscopy (EGD). Patients were identified by querying a billing database with ICD 9 code of 935.1 (Foreign body in the esophagus) and ICD 10 code of T18.128A (Food in esophagus causing other injury, initial encounter), along with endoscopic procedure codes, CPT codes of 43247 (Esophagogastroduodenoscopy procedures), or 43215 (Esophagoscopy, flexible, transoral; with the removal of foreign body) in the same hospital visit. The above search criteria uncovered a total of 455 patients. We reviewed all 455 charts, and 281 patients were excluded based on our exclusion criteria. The final sample size included 174 patients that met our inclusion criteria as shown in Figure 1.

A retrospective analysis was undertaken on relevant clinical data such as patient's age, co-morbidities, type of food, location of food impaction, administration of glucagon, endoscopic technique and complications such as perforations, tears, aspiration and hypoxia.

\section{Inclusion criteria}

We included patients of 18 - 80 years of age who presented to the ED with symptoms of EFI that subsequently underwent EGD in the same hospital visit.

\section{Exclusion criteria}

Patients in whom endoscopy revealed a foreign body other than food or no evidence of food during endoscopy were excluded.

\section{Objectives}

Our primary objective was to determine the risk factors of EFI in patients who presented at SIUH. Secondary objectives included door-to-scope time and complications of food impaction.

\section{Statistical analysis}

Summary of statistics was reported as mean \pm standard deviation or median (first quartile, third quartile) for continuous variables and frequency and percentages for categorical variables. All statistical analyses were performed using the SAS software, Version 9.3 (SAS Inc., Cary, NC, USA).

\section{Results}

Overall, 174 patients had endoscopically confirmed EFI where $102(58.6 \%)$ were males and $72(41.3 \%)$ were females, with a mean age of $61.4 \pm 19$ years. Characteristics of the population and their medical history are shown in Table 1. The type of impacted food was mostly meat in $73(41.9 \%)$ followed by seafood in $11(6.3 \%)$ and vegetables in one $(0.5 \%)$, as shown in Table 2. The most frequent location of food impaction was in the lower one-third of the esophagus in $94(54 \%)$ patients, followed by the upper-third of the esophagus in 35 (20.1\%) cases and middle-third in $25(14.3 \%)$ cases. Glucagon was given to 74 patients $(42.5 \%)$. Amongst those, the median doorto-scope time was $7 \mathrm{~h}$ (range $1.5-24 \mathrm{~h}$ ) in patients who had received glucagon, as opposed to $7 \mathrm{~h}$ (range $1-24 \mathrm{~h}$ ) in those who did not receive it. Possible causes for the EFI included: strictures in $43(24.7 \%)$, Schatzki's rings in 15 (8.6\%), achalasia in eight (4.5\%) and masses in six (3.4\%) (benign in four patients and malignant in two patients) as shown in Table 3. Endoscopic findings included esophagitis, esophageal ulcers, hiatal hernias and Barrett's esophagus, as shown in Table 3. The biopsy was performed in $38(21.8 \%)$ cases during initial endoscopy; on repeat endoscopy, it was done in $12(6.8 \%)$ cases. The push technique was used in $95(54.5 \%)$ patients, and the pull technique was implemented in $83(47.7 \%)$ patients. Endoscopic therapeutic intervention as the first attempt was successful in $165(94.8 \%)$ patients. Nine patients required 
Table 1. Population Characteristics

\begin{tabular}{ll}
\hline Age (years) & $61.4 \pm 19$ \\
Male & $102(58.6 \%)$ \\
Female & $72(41.3 \%)$ \\
Hispanic & $4(2.2 \%)$ \\
Non-Hispanic & $47(27 \%)$ \\
Unknown & $123(70 \%)$ \\
Prior food impaction & $9(5.1 \%)$ \\
Asthma & $15(8.6 \%)$ \\
Diabetes & $20(11.4 \%)$ \\
GERD & $27(15.5 \%)$ \\
Reflux esophagitis & $3(1.7 \%)$ \\
Stricture & $16(9.1 \%)$ \\
Schatzki’s ring & $3(1.7 \%)$ \\
Achalasia & $5(2.8 \%)$ \\
Hiatal hernia & $6(3.4 \%)$ \\
Bariatric surgery & $5(2.8 \%)$ \\
Other GI surgery & $9(5.1 \%)$ \\
\hline
\end{tabular}

Values for categorical variables are shown as frequency (percentage) and for continuous variables as mean \pm standard deviation. GERD: gastroesophageal reflux disease; Gl: gastrointestinal.

a second attempt for removal. Complications were reported in only five $(2.8 \%)$ patients, and these included perforations and tears as shown in Table 4 . Twenty $(11.4 \%)$ patients with food impaction had a history of diabetes mellitus (DM), as shown in Table 1.

\section{Discussion}

Meat or other types of food are the most frequent cause of EFI in adults of the western world. EFI is a common GI emergency that requires timely intervention [7]. The pathophysiology is often attributed to underlying esophageal pathology or the nature of ingested food, or a combination of both factors. In our study, the mean age of our patients with food impaction was $61.4 \pm 19$ years. Most patients who presented with EFI in our study were men $(58.6 \%)$, and this is consistent with the findings from similar studies $[8,9]$. This male pre-dominance might be explained by the increased prevalence of benign esophageal strictures in men [10]. Our study supports this theory
Table 3. Endoscopic Findings

\begin{tabular}{ll}
\hline Endoscopic findings & Number of patients $(\%)$ \\
\hline Esophagitis & $58(33.3 \%)$ \\
Stricture & $43(24.7 \%)$ \\
Hiatal hernia & $29(16.6 \%)$ \\
Ulcer & $16(9.1 \%)$ \\
Ring & $15(8.6 \%)$ \\
Achalasia & $8(4.5 \%)$ \\
Mass & $6(3.4 \%)$ \\
Gastroesophageal reflux disease & $3(1.7 \%)$ \\
Barret's & $2(1.1 \%)$ \\
Other non-specified & $42(24.1 \%)$ \\
\hline
\end{tabular}

as esophageal strictures were the most common cause of EFI in our patient population.

Over the past decade, the prevalence of EFI has increased by more than six-fold [4], affecting younger individuals [8]. This could be partly because of an increase in the prevalence of EoE, a condition that is associated with a high risk for food impaction and is more common in younger patients $[11,12]$. However, our study did not demonstrate any evidence of EoE and this can be explained by the mean age of $61.4 \pm 19$ years in our patient population. Additionally, a lower number of biopsies were obtained during initial and repeat endoscopy.

The lower third of the esophagus was the most common site of EFI, which can easily be explained by the prominent distal anatomic narrowing at the gastroesophageal junction. Previous studies have suggested that esophageal strictures and Schatzki's rings are the leading causes of EFI [4, 5]. Benign peptic strictures and EoE are more common in men [13]. In our study, possible causes of food impaction were strictures in $43(24.7 \%)$, Schatzki's rings in $15(8.6 \%)$, achalasia in eight $(4.5 \%)$ and masses in six (3.4\%) (benign mass in four patients and malignant mass in two patients). Esophagitis in endoscopy was described in $58(33.3 \%)$ cases, but whether this was the cause or the consequence of EFI was not clear.

In our study, $11 \%$ of patients with DM had EFI. As patients with DM suffer from various neuropathy-related complications, esophageal dysmotility is most common [14]. More than $60 \%$ of diabetic patients with peripheral or autonomic neuropathy and an occasional patient without neuropathy have esophageal manometric abnormalities [15]. Based on previous studies, manometric defects associated with DM include hypotensive peristalsis, frequent failed peristalsis, hypotensive

Table 2. Type of Food

\begin{tabular}{|c|c|}
\hline Type of food & Number of patients $(\%)$ \\
\hline Meat & $73(41.9 \%)$ : chicken $19(10.9 \%)$, pork $7(4 \%)$, beef $8(4.5 \%)$, others $39(22.4 \%)$ \\
\hline Vegetarian & $1(0.5 \%)$ \\
\hline Sea food & $11(6.3 \%)$ \\
\hline Fruit & $1(0.5 \%)$ \\
\hline Other than above & $86(49.4 \%)$ \\
\hline
\end{tabular}


Table 4. Characteristics of Patients With Complications

\begin{tabular}{|c|c|c|c|c|c|c|}
\hline & $\begin{array}{l}\text { Age in } \\
\text { years }\end{array}$ & $\begin{array}{l}\text { Compli- } \\
\text { cation }\end{array}$ & Technique & Esophageal pathology & $\begin{array}{l}\text { Door-to-scope } \\
\text { time in hours }\end{array}$ & Underlying medical comorbidities \\
\hline 1 & 107 & Perforation & Pull & Dilated tortuous, spastic esophagus & 24 & Asthma, hypertension, stroke, achalasia \\
\hline 2 & 47 & Tear & Pull & esophagitis & 3 & Iron deficiency anemia \\
\hline 3 & 38 & Perforation & Push & Stricture & 3 & None \\
\hline 4 & 67 & Perforation & Push & Stricture & 5 & Hypertension, anxiety, coronary artery disease \\
\hline 5 & 67 & Tear & Push & Dilated tortuous esophagus & 4 & Achalasia, dyslipidemia, depression \\
\hline
\end{tabular}

lower esophageal sphincter with impaired deglutitive relaxation, simultaneous contractions and repetitive contractions [15]. Theoretically, these abnormalities can pose a significant risk for EFI either independently or in combination with any underlying anatomical abnormality like peptic strictures. Different studies have identified various structural and functional esophageal abnormalities, which may increase the risk of EFI. Still, none of them have investigated if DM can be a potential risk factor for EFI. Even though DM was evident in $11.4 \%$ of patients with EFI, given the lack of a control group, we were unable to ascertain if DM can be an independent risk factor for EFI. Further studies are needed to establish a clear association.

Intravenous glucagon is the frequently used agent for EFI in the ED [16]; glucagon is an endogenous polypeptide secreted from alpha cells in the islets of Langerhans. At pharmacological doses, both $0.25 \mathrm{mg}$ and $0.5 \mathrm{mg}$ have been shown to relax the esophageal smooth muscle and reduce the mean resting pressure of the LES, promoting the spontaneous passage of an impacted food bolus [16]. Higher doses are not more effective in reducing LES pressure [4]. The success rate for the use of glucagon is reportedly between $12 \%$ and $50 \%[6,17]$. However, glucagon is much less effective in patients with underlying structural abnormalities, such as strictures or rings. Hypersensitivity to glucagon and history of pheochromocytoma or insulinoma are contraindications to its administration [18]. Nausea, vomiting and diarrhea are the common side effects [18]. In our study, glucagon was given to $74(42.5 \%)$ patients. Still, it failed to relieve the symptoms of EFI, likely due to multiple factors, namely, the amount of food impacted and anatomical abnormalities in our study population. The mean door-to-scope time was less in patients who had received glucagon. The likely reason for this is that in our study, major reasons for food impaction are strictures, Schatzki's ring, which are structural abnormalities in which glucagon is known to be less effective [9]. This resulted in persistent symptoms of food impaction, prompting to perform earlier endoscopy than the patients who did not receive glucagon.

While the majority of EFI cases resolve spontaneously, $10-20 \%$ of patients require endoscopic intervention [19]. Endoscopic intervention for removal of food impaction includes either the push technique (advancement of the bolus into the stomach) or pull technique (extraction of food) [6]. Pull technique may involve either en bloc removal by using various grasping devices (e.g., polypectomy snare, retrieval net, friction-fit adaptor, or banding cap) or piecemeal. American Soci- ety for Gastrointestinal Endoscopy (ASGE) guidelines previously recommended against push technique because of the risk of perforation; however, two large published case series using the push technique reported no perforations in a total of 375 patients $[2,20]$. Even though there is a risk of perforation in the pull technique when excessive force is applied, it is somewhat safer than the push technique. For an esophageal stricture, it is considered safe to perform dilation after food bolus extraction to reduce the risk of recurrence $[2,20]$. In our study approach to remove the food bolus either by push technique $(95,54.5 \%)$ or pull technique $(83,47.7 \%)$ had no impact on clinical outcomes. Biopsy at the time of EFI is controversial. In our study biopsy was done in $38(21.8 \%)$ cases during initial endoscopy and in $12(6.8 \%)$ cases during repeat endoscopy. We recommend obtaining a biopsy when performing initial endoscopy. If unable to perform an initial biopsy, it should be performed in repeat endoscopy to rule out EoE.

Most common complications of EFI are related to prolonged food impaction that can lead to stasis, ulceration and eventually necrosis with perforation [1]. In our study, a total of five patients had adverse events. Complications due to the push technique were observed in three patients (two perforations and one tear), and for the pull technique, two patients experienced adverse outcomes (one perforation and one tear). Characteristics of patients with complications are mentioned in Table 4. The push technique has been controversial over the years due to the risk of perforation. However, ASGE guidelines and other studies support its efficacy along with other techniques such as en bloc and piecemeal removal [20, 21]. The $94.8 \%$ success rate of removing the EFI during the first endoscopy in our study is similar to that of the most recent studies [4, 22].

Our study's strengths are related to our inclusion criteria; we included patients with endoscopic confirmation of food bolus in the esophagus and evaluated any association of EFI with DM. As a retrospective chart review study, limitations include the lack of blinding of endoscopists and data extraction was limited to the data recorded in the database, and research was done at a single institution, potentially limiting the generalizability of results.

\section{Conclusion}

EFI is more common in males. Esophageal stricture and hiatal hernia were the most common pathologies found in endosco- 
py. Esophagitis was found in $33 \%$ of patients, but we are unable to determine if it is the cause or consequence of EFI. DM was associated with food impaction in only $11 \%$ of patients. However, more studies are needed to determine if DM has any role in EFI. Administration of glucagon resulted in an overall decrease in door-to-scope time. Endoscopy is a safe and effective method for the management of food impaction, and complications reported were minimal.

\section{Learning points}

What is already known: Esophageal stricture and Schatzki's ring are the most common causes of food impaction in adults. Meat is the most common type of food impacted. The endoscopic push technique is the commonly used approach for the removal of food. Complication rates are minimal.

What the new findings are: DM was reported in $11.4 \%$ of patients with EFI. The door-to-scope time was less in patients who received glucagon. The endoscopic push technique appears to be safe with minimal complications.

\section{Acknowledgments}

We want to acknowledge the contributions made by our faculty and staff. Thanks are given to Dr. Reyes, Jonathan, Dr. Acharya, Roshan, and Dr. Rebhan, Anh, for their data collection efforts; given to Dr. Smith, Marianne (Director of Medical Student Education, Assistant professor, Department of Medicine) for valuable suggestions and feedback; given to Ms. Maria A. Farberov (Director, Clinical research programs, Department of Medicine) for guiding the project since the beginning and for her assistance in writing the protocol and designing REDCAP.

\section{Financial Disclosure}

None to declare.

\section{Conflict of Interest}

None to declare.

\section{Informed Consent}

Not applicable.

\section{Author Contributions}

Dr. Polavarapu and Dr. Gumaste designed the project. Dr. Gurala, Dr. Polavarapu, Dr. Philipose collected data. Dr. Demissie analyzed the data and wrote statistical analysis. Dr. Avula, Dr. Idiculla wrote introduction. Dr. Gurala, Dr. Polavarapu, Dr. Philipose, Dr. Amarnath interpreted data, wrote methods, results and discussion. Dr. Gumaste supervised the findings of the work, reviewed and edited the manuscript.

\section{Data Availability}

The authors declare that data supporting the findings of this study are available within the article.

\section{References}

1. Schaefer TJ, Trocinski D. Esophagial Foreign Body. In: StatPearls. Treasure Island (FL), 2021.

2. Longstreth GF, Longstreth KJ, Yao JF. Esophageal food impaction: epidemiology and therapy. A retrospective, observational study. Gastrointest Endosc. 2001;53(2):193198.

3. Lai AT, Chow TL, Lee DT, Kwok SP. Risk factors predicting the development of complications after foreign body ingestion. Br J Surg. 2003;90(12):1531-1535.

4. Sperry SL, Crockett SD, Miller CB, Shaheen NJ, Dellon ES. Esophageal foreign-body impactions: epidemiology, time trends, and the impact of the increasing prevalence of eosinophilic esophagitis. Gastrointest Endosc. 2011;74(5):985-991.

5. Hiremath GS, Hameed F, Pacheco A, Olive A, Davis CM, Shulman RJ. Esophageal food impaction and eosinophilic esophagitis: a retrospective study, systematic review, and meta-analysis. Dig Dis Sci. 2015;60(11):3181-3193.

6. Ko HH, Enns R. Review of food bolus management. Can J Gastroenterol. 2008;22(10):805-808.

7. Melendez-Rosado J, Corral JE, Patel S, Badillo RJ, Francis D. Esophageal food impaction: causes, elective intubation, and associated adverse events. J Clin Gastroenterol. 2019;53(3):179-183.

8. Mahesh VN, Holloway RH, Nguyen NQ. Changing epidemiology of food bolus impaction: is eosinophilic esophagitis to blame? J Gastroenterol Hepatol. 2013;28(6):963-966.

9. Sodeman TC, Harewood GC, Baron TH. Assessment of the predictors of response to glucagon in the setting of acute esophageal food bolus impaction. Dysphagia. 2004;19(1):18-21.

10. Shuja A, Winston DM, Rahman AU, Mitty RD, Jaber BL, Keo T. Esophageal food impaction during cultural holidays and national athletic events. Gastroenterol Rep (Oxf). 2017;5(1):43-46.

11. Mackenzie SH, Go M, Chadwick B, Thomas K, Fang J, Kuwada S, Lamphier S, et al. Eosinophilic oesophagitis in patients presenting with dysphagia - a prospective analysis. Aliment Pharmacol Ther. 2008;28(9):1140-1146.

12. Prasad GA, Alexander JA, Schleck CD, Zinsmeister AR, Smyrk TC, Elias RM, Locke GR, 3rd, et al. Epidemiology of eosinophilic esophagitis over three decades in Olmsted County, Minnesota. Clin Gastroenterol Hepatol. 2009;7(10):1055-1061.

13. Furuta GT, Liacouras CA, Collins MH, Gupta SK, Justinich C, Putnam PE, Bonis P, et al. Eosinophilic esophagitis 
in children and adults: a systematic review and consensus recommendations for diagnosis and treatment. Gastroenterology. 2007;133(4):1342-1363.

14. Gustafsson RJ, Littorin B, Berntorp K, Frid A, Thorsson O, Olsson R, Ekberg O, et al. Esophageal dysmotility is more common than gastroparesis in diabetes mellitus and is associated with retinopathy. Rev Diabet Stud. 2011;8(2):268-275.

15. George NS, Rangan V, Geng Z, Khan F, Kichler A, Gabbard S, Ganocy S, et al. Distribution of esophageal motor disorders in diabetic patients with dysphagia. J Clin Gastroenterol. 2017;51(10):890-895.

16. Alaradi O, Bartholomew M, Barkin JS. Upper endoscopy and glucagon: a new technique in the management of acute esophageal food impaction. Am J Gastroenterol. 2001;96(3):912-913.

17. Desai TK, Stecevic V, Chang CH, Goldstein NS, Badizadegan K, Furuta GT. Association of eosinophilic inflammation with esophageal food impaction in adults. Gastro- intest Endosc. 2005;61(7):795-801.

18. Khayyat YM. Pharmacological management of esophageal food bolus impaction. Emerg Med Int. 2013;2013:924015.

19. Yao CC, Wu IT, Lu LS, Lin SC, Liang CM, Kuo YH, Yang $\mathrm{SC}$, et al. Endoscopic management of foreign bodies in the upper gastrointestinal tract of adults. Biomed Res Int. 2015;2015:658602.

20. Vicari JJ, Johanson JF, Frakes JT. Outcomes of acute esophageal food impaction: success of the push technique. Gastrointest Endosc. 2001;53(2):178-181.

21. Asge Standards of Practice Committee, Ikenberry SO, Jue TL, Anderson MA, Appalaneni V, Banerjee S, Ben-Menachem $\mathrm{T}$, et al. Management of ingested foreign bodies and food impactions. Gastrointest Endosc. 2011;73(6):10851091.

22. Kirchner GI, Zuber-Jerger I, Endlicher E, Gelbmann C, Ott C, Ruemmele P, Scholmerich J, et al. Causes of bolus impaction in the esophagus. Surg Endosc. 2011;25(10):31703174. 